\title{
Pinning controllability of autonomous Boolean control networks
}

\author{
Hongwei $\mathrm{CHEN}^{1}$, Jinling $\mathrm{LIANG}^{1 *}$ \& Zidong $\mathrm{WANG}^{2,3}$ \\ ${ }^{1}$ Department of Mathematics, Southeast University, Nanjing 210096, China; \\ ${ }^{2}$ Department of Computer Science, Brunel University London, Uxbridge, Middlesex UB8 3PH, UK; \\ ${ }^{3}$ Faculty of Engineering, King Abdulaziz University, Jeddah 21589, Saudi Arabia. \\ Received January 1, 2016; accepted xx, 2016; published online xx, 2016
}

\begin{abstract}
Autonomous Boolean networks (ABNs), which are developed to model the Boolean networks (BNs) with regulatory delays, are well known for their advantages of characterizing the intrinsic evolution rules of biological systems such as the gene regulatory networks. As a special type of ABNs with binary inputs, the autonomous Boolean control networks $(\mathrm{ABCNs})$ are introduced for designing and analyzing the therapeutic intervention strategies where the binary inputs represent whether a certain medicine is dominated or not. An important problem in the therapeutic intervention is to design a control sequence steering an $\mathrm{ABCN}$ from an undesirable location (implying a diseased state) to a desirable one (corresponding to a healthy state). Motivated by such background, this paper aims to investigate the reachability and controllability of ABCNs with pinning controllers. Several necessary and sufficient criteria are provided by resorting to the semi-tensor product techniques of matrices. Moreover, an effective pinning control algorithm is presented for steering an ABCN from any given states to the desired state in the shortest time period. Numerical examples are also presented to demonstrate the results obtained.
\end{abstract}

Keywords Autonomous Boolean networks, semi-tensor product, controllability, pinning control scheme, network transition matrix

Citation Chen H W, Liang J L, Wang Z D. Pinning controllability of autonomous Boolean control networks. Sci China Inf Sci, 2016, xx(x): xxxxxx, doi: xxxxxxxxxxxxxx

\section{Introduction}

From a translational perspective, the ultimate objective of genomic research is to uncover the mechanisms with which cells execute and control the enormous number of operations required for normal functions and the ways in which cellular systems fail in disease. A rather wide spectrum of approaches have been developed to model the genetic regulatory networks (GRNs), and the most frequently investigated models include the Boolean model, the Bayesian network model and the differential equations model 1122. Among these models, the Boolean network (BN), originally proposed by Kauffman in 1969 [3], has been proven to be a prominent qualitative tool for modeling the genetic regulatory process. In the past few decades, the study of BNs has received considerable research attention from both the biology and the physics communities. Many excellent results have been available in the literature including the topological structure [4] and the controllability of BNs [5].

* Corresponding author (email: jinlliang@seu.edu.cn) 
In a $\mathrm{BN}$, each gene is approximated as a Boolean node that switches between ON and OFF (1 and 0 , respectively), and the state of a node is updated at discrete time instants according to a pre-assigned logic function which depends on the states of that node's inputs on the previous time instant. As pointed out in 6], many biological systems have exogenous perturbations that can be described as 'controls', and the concept of Boolean control networks (BCNs) has been put forward by adding binary inputs. For instance, when modeling the progression of a disease by BCNs, the binary input may represent whether a certain medicine is administered or not at each time instant.

Actually, a BCN can be regarded as a family of BNs in which the value of the control determines which $\mathrm{BN}$ is active. In this setup, the interest in the control problem for BCNs corresponding to therapeutic interventions arises primarily in the field of systems biology. However, due to the lack of effective tools to deal with logical systems, no unified criterion has been available for testing the controllability until the introduction of the semi-tensor product (STP) method originally proposed in [7]. Thanks to this novel STP technique, the logical dynamics of a BN $(\mathrm{BCN})$ can be uniquely transformed into a standard discretetime linear (bilinear) dynamical system. Consequently, several analysis and control problems, which include but are not limited to, controllability and observability 8 10, stability and stabilization 11] 14, optimal control [15, 16, system decomposition [17, 18, have been extensively investigated in recent years. For more details about the STP, we refer the readers to [19,20].

On the other hand, time delays in genetic regulatory process are inevitable due primarily to the slow processes of transcription, translation and translocation or the finite switching speed of amplifiers. It has now been well recognized that time delays in GRNs may play an important role in the predictions of the dynamics of mRNA and protein concentrations, and theoretical models without consideration of these delay factors may even have led to wrong predictions 21. As such, when modeling the dynamic behaviors of GRNs by BCNs, the inherent time delays should be taken into account. Note that the controllability issue for $\mathrm{BCNs}$ with time delays has emerged as a research topic of great importance. In [22, the controllability of BCNs with time-invariant delays in states has been studied by increasing the dimension of the state space. This approach has been further adopted in 23 and 24 to deal with the controllability of higher-order BCNs. In [25, the authors have considered the controllability of timevariant $\mathrm{BCNs}$ as well as BCNs with multiple time-variant bounded state delays. An equivalent test criterion for the controllability of BCNs with unbounded time-delays in states has been given in [26] based on a new proposed concept called controllability constructed path.

A recent yet significant discovery in the cellular reprogramming field is that full control and reprogramming of biological systems may be achieved by controlling only a few key factors [27. This discovery seems to be in contradiction to the conventional definition of controllability of BCNs that concerns with the control of all the system's nodes. As a matter of fact, for a large-scale GRN, it is usually difficult to add controllers to all nodes. In order to reduce the number of controller, a natural idea is to control the network by pining only part of the nodes. In [28, a BN model has been developed to reproduce the two-phase dynamics of the p53 network in response to DNA damage, where a practical control scheme has been proposed by pinning the state of a critical node to steer the system to the desired attractor pertaining to the desired final state. It is worth emphasizing that such kind of pinning control scheme is of paramount importance for medical treatment and genetic engineering. Recently, by resorting to the STP technique, the pinning controllability of BCNs has been investigated in 29] and the pinning control design for stabilizing the BNs has been given in 30 .

In 31, Ghil and Mullhaupt have introduced the Boolean delay equations as an autonomous BN $(\mathrm{ABN})$, and this model has then been widely applied to yeast cell cycle and electronic circuits 32 . In [33, based on the logic gates, Rivera-Durón has built an electronic realization of an ABN of five nodes with external Boolean signal that can be regard as control input, namely autonomous Boolean control network (ABCN) (see Example 3 for reference), and the forced synchronization issue of two identical ABNs (forced by a common external signal) has been addressed. The recent work in 34 has shown that the analysis on complex dynamics in an electronic circuit is beneficial for capturing the qualitative aspects of the structure and dynamics of GRNs. Very recently, Cheng et al. have first presented the ABN framework in mathematical terms, described the biological meaning of its time-delay parameters [35], and then applied it to the Drosophila segment polarity gene regulatory system. Experimental results have further confirmed that important timing information associated with the regulatory interactions among genes can be faithfully represented in ABN models, and that such models can provide a direct insight into understanding and controlling the GRNs. All the above theoretical and experimental results indicate that the $\mathrm{ABCN}$ is an important and appropriate model to simulate and analyze the GRNs. 
Chen H W, et al. Sci China Inf Sci January 2016 Vol. xx xxxxxx:3

In this paper, by following the main stream of research, we further consider the pining controllability problem of ABCNs in which only a selected fraction of nodes (not every node of the ABCNs) are controlled. Based on the algebraic representation of logical dynamics in terms of STP of matrices, the inherent special structures of the network transition matrix are investigated, and matrix testing criteria for the pinning controllability of ABCNs are obtained. Then, we further devise practical control schemes for steering an ABCN between two given states in a given number of time-steps by pinning a selected fraction of nodes. The approach proposed offers insights into understanding and controlling the practical biological systems, which is of paramount importance for the therapeutic intervention and in the genetic engineering.

The rest of the paper is organized as follows. Section 2 contains some notations and preliminaries on the STP of matrices. The main results of this paper are presented in Section 3, and a brief conclusion is drawn in Section 4.

\section{Preliminaries}

The following notations will be used throughout this paper.

- $\mathcal{Z}$ and $\mathcal{N}$ are the sets of integers and nonnegative integers, respectively.

- $\mathcal{D}:=\{1,0\}$, and $\mathcal{D}^{n}=\underbrace{\mathcal{D} \times \cdots \times \mathcal{D}}_{n}$.

- $\mathbb{R}^{m \times n}$ means the set of all $m \times n$ real matrices.

- $\Delta_{m}:=\left\{\delta_{m}^{1}, \delta_{m}^{2}, \ldots, \delta_{m}^{m}\right\}$, where $\delta_{m}^{i}$ is the $i$ th column of the identity matrix $I_{m}$ for $i=1,2, \ldots, m$.

- A matrix $A \in \mathbb{R}^{m \times n}$ is called a logical matrix if $A=\left[\delta_{m}^{i_{1}} \delta_{m}^{i_{2}} \cdots \delta_{m}^{i_{n}}\right]\left(i_{1}, i_{2}, \ldots, i_{n} \in\{1,2, \ldots, m\}\right)$, which is also expressed by $A=\delta_{m}\left[i_{1}, i_{2}, \ldots, i_{n}\right]$ for simplicity. The set of all $m \times n$ logical matrices is denoted by $\mathcal{L}_{m \times n}$.

- $\operatorname{Col}_{i}(A)$ (respectively, $\operatorname{Row}_{i}(A)$ ) is used to represent the $i$ th column (respectively, row) of matrix $A$.

- $\operatorname{Blk}_{i}(A)$ represents the $i$ th block of matrix $A=\left[\begin{array}{llll}A_{1} & A_{2} & \cdots & A_{p}\end{array}\right]$, where all $A_{i}(i=1,2, \ldots, p)$ have the same dimensions.

- $\mathbf{1}_{m}$ denotes the $m$-dimensional column vector with all entries being 1, i.e., $\mathbf{1}_{m}=\sum_{k=1}^{m} \delta_{m}^{k}$, and $\mathbf{1}_{m}^{\mathrm{T}}=\left[\begin{array}{llll}1 & 1 & \cdots & 1\end{array}\right]$, where ' $\mathrm{T}$ ' represents the transpose of $\mathbf{1}_{m}$.

- For $A \in \mathbb{R}^{m \times n}$ and $B \in \mathbb{R}^{r \times n}$, the Khatri-Rao product of $A$ and $B$, denoted by $A * B$, is defined as $A * B=\left[\operatorname{Col}_{1}(A) \otimes \operatorname{Col}_{1}(B) \cdots \operatorname{Col}_{n}(A) \otimes \operatorname{Col}_{n}(B)\right]$, where ' $\otimes$ ' is the Kronecker product.

Firstly, the definition and some basic properties of STP are introduced that are useful in our later discussion.

Definition 1 ( [7]). The STP of two matrices $A \in \mathbb{R}^{m \times n}$ and $B \in \mathbb{R}^{p \times q}$ is defined as

$$
A \ltimes B=\left(A \otimes I_{\alpha / n}\right)\left(B \otimes I_{\alpha / p}\right),
$$

where $\alpha=\operatorname{lcm}(n, p)$ is the least common multiple of $n$ and $p$.

Remark 1. When $n=p, A \ltimes B=\left(A \otimes I_{1}\right)\left(B \otimes I_{1}\right)=A B$. Therefore, the STP is a generalization of the conventional matrix product that provides a way to make two matrices with arbitrary dimensions multiplicable. Hereafter, we simply call it 'product' and omit the symbol ' $\alpha$ ' if no confusion arises.

Definition 2 ( [7]). A swap matrix $W_{[m, n]}$ is an $m n \times m n$ matrix defined as follows: its rows and columns are labeled by double index $(i, j)$, where the columns are arranged by the ordered multi-index $I d(i, j ; m, n)$ and the rows are arranged by the ordered multi-index $\operatorname{Id}(j, i ; n, m)$. The element at the position $((I, J),(i, j))$ is then set to

$$
w_{(I, J),(i, j)}=\delta_{i, j}^{I, J}= \begin{cases}1, & I=i \text { and } J=j, \\ 0, & \text { otherwise. }\end{cases}
$$

Lemma 1 ( [7]). The STP of matrices has the following properties:

1) Let $X \in \Delta_{m}$ and $Y \in \Delta_{n}$, then $Y \ltimes X=W_{[m, n]} \ltimes X \ltimes Y$, where $W_{[m, n]}$ is the swap matrix.

2) Let the $k^{2} \times k$ logical matrix $\Psi_{k}=\left[\delta_{k}^{1} \otimes \delta_{k}^{1} \delta_{k}^{2} \otimes \delta_{k}^{2} \cdots \delta_{k}^{k} \otimes \delta_{k}^{k}\right]$ and $Z \in \Delta_{k}$, then $Z \ltimes Z=\Psi_{k} Z$.

Secondly, by identifying $1 \sim \delta_{2}^{1}$ and $0 \sim \delta_{2}^{2}$, where ' $\sim$ ' means two different/equivalent forms of the same object, the logical variable in $\mathcal{D}$ then takes value from $\Delta_{2}$. And then a logical function with $n$ variables in $\mathcal{D}$ can be expressed in the algebraic form as follows. 
Chen H W, et al. Sci China Inf Sci January 2016 Vol. xx xxxxxx:4

Lemma 2 ( [7]). Let $f\left(x_{1}, x_{2}, \ldots, x_{n}\right): \mathcal{D}^{n} \rightarrow \mathcal{D}$ be a logical function. Then there exists a unique matrix $M_{f} \in \mathcal{L}_{2 \times 2^{n}}$, called the structure matrix of $f$, such that

$$
f\left(x_{1}, x_{2}, \ldots, x_{n}\right)=M_{f} \ltimes_{i=1}^{n} x_{i}, \quad x_{i} \in \Delta_{2}
$$

where $\ltimes_{i=1}^{n} x_{i}=x_{1} \ltimes x_{2} \ltimes \cdots \ltimes x_{n}$.

Finally, to proceed, some properties of the Kronecker product are presented which will be used in the sequel.

Lemma 3. The following results hold for the Kronecker product of matrices:

1) If matrices $A, B, C$ and $D$ have proper dimensions, then

$$
(A \otimes B)(C \otimes D)=(A C) \otimes(B D) .
$$

2) If $X \in \Delta_{m}, Y \in \Delta_{n}$, then $\mathbf{1}_{m}^{\mathrm{T}} X=1, \mathbf{1}_{n}^{\mathrm{T}} Y=1, X \ltimes Y=X \otimes Y$ and

$$
X=\left(I_{m} X\right) \otimes\left(\mathbf{1}_{n}^{\mathrm{T}} Y\right)=\left(I_{m} \otimes \mathbf{1}_{n}^{\mathrm{T}}\right) X Y, \quad Y=\left(\mathbf{1}_{m}^{\mathrm{T}} X\right) \otimes\left(I_{n} Y\right)=\left(\mathbf{1}_{m}^{\mathrm{T}} \otimes I_{n}\right) X Y .
$$

\section{Main results}

\subsection{Algebraic representation form of the $\mathrm{ABCNs}$}

A conventional BCN with $m$ controllers and $n$ nodes can be described by the following discrete-time logical dynamic system

$$
x_{i}(t+1)=f_{i}\left(u_{1}(t), \ldots, u_{m}(t), x_{1}(t), \ldots, x_{n}(t)\right), \quad i=1, \ldots, n
$$

where $x_{i}$ and $u_{j}(j=1,2, \ldots, m)$ are the $i$ th state variable and the control input variables taking values in $\mathcal{D}, f_{i}: \mathcal{D}^{m+n} \rightarrow \mathcal{D}(i=1, \ldots, n)$ are the Boolean functions, $t \in \mathcal{Z}$ satisfies $t \geqslant t_{0}$ with $t_{0} \in \mathcal{Z}$ being the initial time.

In many cases of interest, signals propagate in the network with such a slow speed so that the time delays along the links are comparable to or larger than the characteristic response times of the nodes. In this case, time delays must be introduced in the model which can be described by a set of numbers $\left\{\tau_{i j} \in \mathcal{N}: i, j=1, \ldots, n\right\}$, where $\tau_{i j}$ is the time for node $x_{j}$ to have an effect on node $x_{i}$, i.e., the time that a signal takes to propagate to node $i$ from node $j$. And the delayed feedbacks among the Boolean variables are characterized by the following ABCNs:

$$
x_{i}(t+1)=f_{i}\left(u_{1}(t), \ldots, u_{m}(t), x_{1}\left(t-\tau_{i 1}\right), \ldots, x_{n}\left(t-\tau_{i n}\right)\right), \quad i=1, \ldots, n .
$$

For example, in GRNs, time delays are ubiquitous due to the slow biochemical reactions in the process of transcription, translation, and degradation. And the recent publication [35] shows that the ABCN is just an important and appropriate model to simulate and analyze the dynamics of GRNs.

On the other hand, for some special cases of biological systems such as mammalian cells, full control effect might also be achieved by controlling only a few key factors [27, which seems to conflict with the case for the conventional ABCNs where all nodes are required to exert full control. This demonstrates that when investigating the biological systems researches on the pinning control of ABCNs are not only meaningful but also necessary. Based on the above discussions, in this paper, we consider the pinning controllability of ABCNs with $n$ nodes, where the nodes $i_{1}, i_{2}, \ldots, i_{r}(1 \leqslant r<n)$ are selected to be controled. Without loss of generality, we assume that $i_{k}=k(k=1,2, \ldots, r)$. Then, the dynamics of ABCNs with $r$ pinning controllers can be described as follows:

$$
\left\{\begin{aligned}
x_{1}(t+1) & =f_{1}\left(u_{1}(t), x_{1}\left(t-\tau_{11}\right), \ldots, x_{n}\left(t-\tau_{1 n}\right)\right), \\
& \vdots \\
x_{r}(t+1) & =f_{r}\left(u_{r}(t), x_{1}\left(t-\tau_{r 1}\right), \ldots, x_{n}\left(t-\tau_{r n}\right)\right), \\
x_{r+1}(t+1) & =f_{r+1}\left(x_{1}\left(t-\tau_{r+1,1}\right), \ldots, x_{n}\left(t-\tau_{r+1, n}\right)\right), \\
& \vdots \\
x_{n}(t+1) & =f_{n}\left(x_{1}\left(t-\tau_{n 1}\right), \ldots, x_{n}\left(t-\tau_{n n}\right)\right) .
\end{aligned}\right.
$$


Let $x=\ltimes_{k=1}^{n} x_{k}$ and $\tau=\max _{1 \leqslant i, j \leqslant n}\left\{\tau_{i j}\right\}$, combining with Lemmas 1, 3ystem (3) can be transformed into the component-wise algebraic form as follows:

$$
\begin{aligned}
& x_{i}(t+1)=\hat{F}_{i} u_{i}(t) x(t) \cdots x(t-\tau), \quad i=1, \ldots, r \\
& x_{j}(t+1)=\hat{F}_{j} x(t) \cdots x(t-\tau), \quad j=r+1, \ldots, n
\end{aligned}
$$

where $\hat{F}_{i} \in \mathcal{L}_{2 \times 2^{n(\tau+1)+1}}$ and $\hat{F}_{j} \in \mathcal{L}_{2 \times 2^{n(\tau+1)}}$. By further denoting $x^{1}=\ltimes_{k=1}^{r} x_{k}, x^{2}=\ltimes_{k=r+1}^{n} x_{k}$ and $u=\ltimes_{k=1}^{r} u_{k}$, it follows from (4) that

$$
\begin{aligned}
& x^{1}(t+1)=F_{1} u(t) x(t) \cdots x(t-\tau), \\
& x^{2}(t+1)=F_{2} x(t) \cdots x(t-\tau),
\end{aligned}
$$

where $F_{1}=\left(\otimes_{k=1}^{r} \hat{F}_{k}\right) \ltimes_{k=1}^{r-1}\left[\left(I_{2^{k}} \otimes W_{\left[2,2^{n(\tau+1)}\right]}\right)\left(I_{2^{k+1}} \otimes \Psi_{\left.2^{n(\tau+1)}\right)}\right)\right] \in \mathcal{L}_{2^{r} \times 2^{n(\tau+1)+r}}$ and $F_{2}=*_{k=r+1}^{n} \hat{F}_{k} \in$ $\mathcal{L}_{2^{n-r} \times 2^{n(\tau+1)}}$ in which ' $*$ ' is the Khatri-Rao product. By (5b) and Lemma 3, one obtains

$$
\begin{aligned}
x^{2}(t+1) & =F_{2}\left(\mathbf{1}_{2^{r}}^{\mathrm{T}} \otimes I_{2^{n(\tau+1)}}\right) u(t) x(t) \cdots x(t-\tau) \\
& =\left(\mathbf{1}_{2^{r}}^{\mathrm{T}} \otimes F_{2}\right) u(t) x(t) \cdots x(t-\tau) \\
& \triangleq \bar{F}_{2} u(t) x(t) \cdots x(t-\tau),
\end{aligned}
$$

where $\bar{F}_{2}=\left(\mathbf{1}_{2^{r}}^{\mathrm{T}} \otimes F_{2}\right) \in \mathcal{L}_{2^{n-r} \times 2^{n(\tau+1)+r}}$. This together with (5a) gives the following algebraic form of system (3):

$$
x(t+1)=F u(t) x(t) \cdots x(t-\tau),
$$

where $F=F_{1} * \bar{F}_{2} \in \mathcal{L}_{2^{n} \times 2^{n(\tau+1)+r}}$, called the network transition matrix of ABCN (3).

\subsection{Structure of the network transition matrix}

In the following, we further investigate the inherent special structure of the network transition matrix $F$ in the algebraic equation (7). Suppose that $F_{1}=\delta_{2^{r}}\left[i_{1}, i_{2}, \ldots, i_{2^{n(\tau+1)+r}}\right]$ and $F_{2}=\delta_{2^{n-r}}\left[j_{1}, j_{2}, \ldots, j_{2^{n(\tau+1)}}\right]$, one has $\bar{F}_{2}=[\underbrace{F_{2} \cdots F_{2}}_{2^{r}}]$. Note that $F=F_{1} * \bar{F}_{2}$, it thus follows that

$$
\begin{aligned}
F=\delta_{2^{n}} & {\left[\left(i_{1}-1\right) 2^{n-r}+j_{1}, \ldots,\left(i_{2^{n(\tau+1)}}-1\right) 2^{n-r}+j_{2^{n(\tau+1)}},\right.} \\
& \left(i_{2^{n(\tau+1)}+1}-1\right) 2^{n-r}+j_{1}, \ldots,\left(i_{2^{n(\tau+1)} \cdot 2}-1\right) 2^{n-r}+j_{2^{n(\tau+1)}}, \\
& \cdots \\
& \left.\left(i_{2^{n(\tau+1)} \cdot\left(2^{r}-1\right)+1}-1\right) 2^{n-r}+j_{1}, \ldots,\left(i_{2^{n(\tau+1)} \cdot 2^{r}}-1\right) 2^{n-r}+j_{2^{n(\tau+1)}}\right] .
\end{aligned}
$$

Theorem 1. Let (7) be the algebraic representation of $\mathrm{ABCN}$ (3). Then the network transition matrix for the ABCN (3) must be in the form of (8).

Example 1. Consider the following $\mathrm{ABCN}$ with three nodes and two pinning controllers:

$$
\left\{\begin{array}{l}
x_{1}(t+1)=u_{1}(t) \leftrightarrow\left[x_{1}(t) \vee x_{2}(t-1)\right], \\
x_{2}(t+1)=u_{2}(t) \vee x_{3}(t-2), \\
x_{3}(t+1)=x_{1}(t) \wedge x_{2}(t-1),
\end{array}\right.
$$

where ' $\leftrightarrow$ ', ' $\vee$ ', and ' $\wedge$ ' represent the logical functions XNOR, OR, and AND, respectively. Denote $x^{1}=\ltimes_{k=1}^{2} x_{k}, x^{2}=x_{3}, x=\ltimes_{k=1}^{3} x_{k}$, and $u=\ltimes_{k=1}^{2} u_{k}$, by resorting to the STP technique, one can obtain the following algebraic representation

$$
\left\{\begin{array}{l}
x^{1}(t+1)=F_{1} u(t) x(t) x(t-1) x(t-2), \\
x^{2}(t+1)=F_{2} x(t) x(t-1) x(t-2),
\end{array}\right.
$$

where $F_{1}=\delta_{4}[\underbrace{1,1, \ldots, 3}_{2^{9}}, \underbrace{1,2, \ldots, 4}_{2^{9}}, \underbrace{3,3, \ldots, 1}_{2^{9}}, \underbrace{1,3, \ldots, 2}_{2^{9}}]$ and $F_{2}=\delta_{2}[\underbrace{1,1, \ldots, 2}_{2^{9}}]$. Then the network transition matrix $F$ can be calculated as follows

$$
\begin{aligned}
F=\delta_{8}[ & (1-1) 2+1,(1-1) 2+1, \ldots,(3-1) 2+2, \\
& (1-1) 2+1,(2-1) 2+1, \ldots,(4-1) 2+2, \\
& (3-1) 2+1,(3-1) 2+1, \ldots,(1-1) 2+2, \\
& (1-1) 2+1,(3-1) 2+1, \ldots,(2-1) 2+2],
\end{aligned}
$$


Chen H W, et al. Sci China Inf Sci January 2016 Vol. xx xxxxxx:6

which is just in the form of (8).

Now, we consider the following type of ABCN with $n$ nodes and $s$ nodes $(1 \leqslant s<n)$ are selected to be controlled. Without loss of generality, the first $s$ nodes are assumed to be controlled:

$$
\left\{\begin{aligned}
x_{1}(t+1) & =g_{1}\left(u_{1}(t), \ldots, u_{r}(t), x_{1}\left(t-\tau_{11}\right), \ldots, x_{n}\left(t-\tau_{1 n}\right)\right) \\
& \vdots \\
x_{s}(t+1) & =g_{s}\left(u_{1}(t), \ldots, u_{r}(t), x_{1}\left(t-\tau_{s 1}\right), \ldots, x_{n}\left(t-\tau_{s n}\right)\right) \\
x_{s+1}(t+1) & =g_{s+1}\left(x_{1}\left(t-\tau_{s+1,1}\right), \ldots, x_{n}\left(t-\tau_{s+1, n}\right)\right) \\
& \vdots \\
x_{n}(t+1) & =g_{n}\left(x_{1}\left(t-\tau_{n 1}\right), \ldots, x_{n}\left(t-\tau_{n n}\right)\right)
\end{aligned}\right.
$$

Similar to system (3), denoting $x^{1}=\ltimes_{k=1}^{s} x_{k}, x^{2}=\ltimes_{k=s+1}^{n} x_{k}, x=\ltimes_{k=1}^{n} x_{k}$, and $u=\ltimes_{k=1}^{r} u_{k}$, a direct computation using the properties of STP can easily produce the following algebraic representation of system (11):

$$
\begin{array}{r}
x^{1}(t+1)=G_{1} u(t) x(t) \cdots x(t-\tau), \\
x^{2}(t+1)=G_{2} x(t) \cdots x(t-\tau) .
\end{array}
$$

Let $G_{1}=\delta_{2^{s}}\left[i_{1}, i_{2}, \ldots, i_{2^{n(\tau+1)+r}}\right]$ and $G_{2}=\delta_{2^{n-s}}\left[j_{1}, j_{2}, \ldots, j_{2^{n(\tau+1)}}\right]$, an algebraic form of system (11) can be obtained as

$$
x(t+1)=G u(t) x(t) \cdots x(t-\tau),
$$

where the transition matrix $G$ satisfies the following form:

$$
\begin{aligned}
G=\delta_{2^{n}}[ & \left(i_{1}-1\right) 2^{n-s}+j_{1}, \ldots,\left(i_{2^{n(\tau+1)}}-1\right) 2^{n-s}+j_{2^{n(\tau+1)}}, \\
& \left(i_{2^{n(\tau+1)}+1}-1\right) 2^{n-s}+j_{1}, \ldots,\left(i_{2^{n(\tau+1)} \cdot 2}-1\right) 2^{n-s}+j_{2^{n(\tau+1)}}, \\
& \cdots \\
& \left.\left(i_{2^{n(\tau+1)} \cdot\left(2^{r}-1\right)+1}-1\right) 2^{n-s}+j_{1}, \ldots,\left(i_{2^{n(\tau+1)} \cdot 2^{r}}-1\right) 2^{n-s}+j_{2^{n(\tau+1)}}\right] .
\end{aligned}
$$

Corollary 1. Let (13) be the algebraic representation of ABCN (11). Then the network transition matrix of the system (11) must be in the form of (14).

Comparing Theorem 1 with Corollary 1, one can find that the network transition matrix $G$ has the same form with that of $F$ when $s=r$. In other words, if $s=r$, then systems (3) and (11) have the same structural algebraic representation (77), where $G$ in (14) is in the same form of (8). Therefore, for a given network transition matrix in the form of (8), one might need to judge whether the dynamic of the logical network is expressed in the framework of (3) or (11). In the following, we will investigate this problem. To this end, we first define a set of logical matrices $S_{i}^{r} \in \mathcal{L}_{2 \times 2^{r}}$, called retrievers, as follows

$$
\begin{aligned}
S_{i}^{r} & =\delta_{2}[\underbrace{1, \ldots, 1}_{2^{r-i}}, \underbrace{2, \ldots, 2}_{2^{r-i}}, \ldots, \underbrace{1, \ldots, 1}_{2^{r-i}}, \underbrace{2, \ldots, 2}_{2^{r-i}}] \\
& \triangleq\left[\operatorname{Blk}_{1}\left(S_{i}^{r}\right) \operatorname{Blk}_{2}\left(S_{i}^{r}\right) \cdots \operatorname{Blk}_{2^{i}}\left(S_{i}^{r}\right)\right], \quad i=1, \ldots, r
\end{aligned}
$$

where $\operatorname{Blk}_{p}\left(S_{i}^{r}\right)=\delta_{2}[\underbrace{1, \ldots, 1}_{2^{r-i}}]$ for $p \in \mathbf{p}:=\left\{1,3, \ldots, 2^{i}-1\right\}$ and $\operatorname{Blk}_{q}\left(S_{i}^{r}\right)=\delta_{2}[\underbrace{2, \ldots, 2}_{2^{r-i}}]$ for $q \in \mathbf{q}:=$ $\left\{2,4, \ldots, 2^{i}\right\}$.

The algebraic representation (12b) shows that $x^{2}(t+1)$ is not affected by the control $u(t)$, hence one just need to consider $x^{1}(t+1)$. Note that $S_{i}^{r} x^{1}=x_{i}(i=1, \ldots, r)$, from (12a) we obtain

$$
\begin{aligned}
x_{i}(t+1) & =S_{i}^{r} G_{1} u(t) x(t) \cdots x(t-\tau) \\
& \triangleq \tilde{G}_{i} u(t) x(t) \cdots x(t-\tau),
\end{aligned}
$$


where $\tilde{G}_{i}=S_{i}^{r} G_{1} \in \mathcal{L}_{2 \times 2^{n(\tau+1)+r}}$. If the component-wise algebraic form (16) of the network (11) has the same form with (4a), then there exists matrix $\hat{G}_{i} \in \mathcal{L}_{2 \times 2^{n(\tau+1)+1}}$ such that

$$
\begin{aligned}
x_{i}(t+1) & =\tilde{G}_{i} u(t) x(t) \cdots x(t-\tau) \\
& =\hat{G}_{i} u_{i}(t) x(t) \cdots x(t-\tau) \\
& =\hat{G}_{i} S_{i}^{r} u(t) x(t) \cdots x(t-\tau) .
\end{aligned}
$$

Since $\hat{G}_{i}(i=1, \ldots, r)$ are $2 \times 2^{n(\tau+1)+1}$ logical matrices, we split each of them into 2 equal blocks as $\hat{G}_{i}=\left[\hat{G}_{i 1} \hat{G}_{i 2}\right]$, where $\hat{G}_{i k}=\operatorname{Blk}_{k}\left(\hat{G}_{i}\right) \in \mathcal{L}_{2 \times 2^{n(\tau+1)}}, k=1,2$. It follows from the above equation that

$$
\begin{aligned}
\tilde{G}_{i} & =\hat{G}_{i} S_{i}^{r} \\
& =\left[\hat{G}_{i 1} \hat{G}_{i 2}\right] \delta_{2}[\underbrace{1, \ldots, 1}_{2^{r-i}}, \underbrace{2, \ldots, 2}_{2^{r-i}}, \cdots, \underbrace{1, \ldots, 1}_{2^{r-i}}, \underbrace{2, \ldots, 2}_{2^{r-i}}] \\
& =[\underbrace{\hat{G}_{i 1} \cdots \hat{G}_{i 1}}_{2^{r-i}} \underbrace{\hat{G}_{i 2} \cdots \hat{G}_{i 2}}_{2^{r-i}} \cdots \underbrace{\hat{G}_{i 1} \cdots \hat{G}_{i 1}}_{2^{r-i}} \underbrace{\hat{G}_{i 2} \cdots \hat{G}_{i 2}}_{2^{r-i}}] \\
& \triangleq\left[\operatorname{Blk}_{1}\left(\tilde{G}_{i}\right) \operatorname{Blk}_{2}\left(\tilde{G}_{i}\right) \cdots \operatorname{Blk}_{2^{i}}\left(\tilde{G}_{i}\right)\right],
\end{aligned}
$$

which implies the following result about the inherent special structure of the structure matrix $\tilde{G}_{i}$.

Theorem 2. Let (13) be the algebraic representation of ABCN (11), where $G$ is in the form of (8) (i.e., $r=s$ ). Then one can obtain matrices $G_{1} \in \mathcal{L}_{2^{s} \times 2^{n(\tau+1)+r}}, G_{2} \in \mathcal{L}_{2^{n-s} \times 2^{n(\tau+1)}}$, and $\tilde{G}_{i} \in \mathcal{L}_{2 \times 2^{n(\tau+1)+r}}$, $i=1, \ldots, r$, satisfying $G=G_{1} *\left(\mathbf{1}_{2^{r}}^{\mathrm{T}} \otimes G_{2}\right)$ and $G_{1}=\tilde{G}_{1} * \cdots * \tilde{G}_{r}$. Furthermore, if there exists matrix $\hat{G}_{i}=\left[\hat{G}_{i 1} \hat{G}_{i 2}\right] \triangleq\left[\operatorname{Blk}_{1}\left(\hat{G}_{i}\right) \operatorname{Blk}_{2}\left(\hat{G}_{i}\right)\right]$ such that $\operatorname{Blk}_{p}\left(\tilde{G}_{i}\right)=\mathbf{1}_{2^{r-i}}^{\mathrm{T}} \otimes \hat{G}_{i 1}$ for $p \in \mathbf{p}$ and $\operatorname{Blk}_{q}\left(\tilde{G}_{i}\right)=$ $\mathbf{1}_{2^{r-i}}^{\mathrm{T}} \otimes \hat{G}_{i 2}$ for $q \in \mathbf{q}$, which $i=1, \ldots, r$, then the dynamic of the time delay BCN (11) is in the form of (3).

Remark 2. In fact, if the matrix $\tilde{G}_{i}$ is in the form of (18), then one can easily derive the component-wise algebraic form of $\mathrm{ABCN}(3)$ as follows:

$$
\left\{\begin{aligned}
x_{1}(t+1) & =\hat{G}_{1} u_{1}(t) x(t) \cdots x(t-\tau), \\
& \vdots \\
x_{r}(t+1) & =\hat{G}_{r} u_{r}(t) x(t) \cdots x(t-\tau), \\
x_{r+1}(t+1) & =\hat{G}_{r+1} x(t) \cdots x(t-\tau), \\
& \vdots \\
x_{n}(t+1) & =\hat{G}_{n} x(t) \cdots x(t-\tau),
\end{aligned}\right.
$$

where $\hat{G}_{r+1} * \cdots * \hat{G}_{n}=G_{2}$. Based on (19), one can reconstruct the ABCN (3) in its logical form by resorting to the method proposed in 8 .

Example 2. Suppose that the algebraic representation of an $A B C N$ is

$$
x(t+1)=G u(t) x(t) x(t-1) x(t-2),
$$

where $x=\ltimes_{k=1}^{3} x_{k}, u=u_{1} u_{2}$, and the network transition matrix $G$ is given the same as presented in (10). Then one has matrices $G_{1}=F_{1}$ and $G_{2}=F_{2}$ satisfying $G=G_{1} *\left(\mathbf{1}_{2^{2}}^{\mathrm{T}} \otimes G_{2}\right)$. Furthermore, by calculation, we can find two matrices $\tilde{G}_{1}$ and $\tilde{G}_{2}$ such that $G_{1}=\tilde{G}_{1} * \tilde{G}_{2}$ :

$$
\left\{\begin{aligned}
\tilde{G}_{1} & =\delta_{2}[\underbrace{1,1, \ldots, 2}_{2^{9}}, \vdots \underbrace{1,1, \ldots, 2}_{2^{9}}, \vdots \underbrace{2,2, \ldots, 1}_{2^{9}}, \vdots \underbrace{2,2, \ldots, 1}_{2^{9}}], \\
& \triangleq\left[\hat{G}_{11} \hat{G}_{11} \hat{G}_{12} \hat{G}_{12}\right], \\
\tilde{G}_{2} & =\delta_{2}[\underbrace{1,1, \ldots, 1}_{2^{9}}, \vdots \underbrace{1,2, \ldots, 2}_{2^{9}}, \vdots \underbrace{1,1, \ldots, 1}_{2^{9}}, \vdots \underbrace{1,2, \ldots, 2}_{2^{9}}], \\
& \triangleq\left[\hat{G}_{21} \hat{G}_{22} \hat{G}_{21} \hat{G}_{22}\right] .
\end{aligned}\right.
$$


Chen H W, et al. Sci China Inf Sci January 2016 Vol. xx xxxxxx:8

Therefore, one can conclude that the dynamic of this $\mathrm{ABCN}$ is in the form of (3) by Theorem2, Moreover, the dynamical model of this $\mathrm{ABCN}$ can be constructed in its logical form as (9).

\subsection{Equivalent form of the $\mathrm{ABCNs}$}

Denote $z_{k}(t)=x(t-k)(k=0,1, \ldots, \tau)$ and $z=\ltimes_{k=0}^{\tau} z_{k}$, the algebraic form (7) can be converted into the form

$$
x(t+1)=F u(t) z(t) .
$$

This, together with Lemma 3 yields

$$
\begin{aligned}
z(t+1) & =x(t+1) x(t) \cdots x(t-\tau+1) \\
& =\left(I_{2^{n(\tau+1)}} \otimes \mathbf{1}_{2^{n}}^{\mathrm{T}}\right) x(t+1) x(t) \cdots x(t-\tau+1) x(t-\tau) \\
& =\left(I_{2^{n(\tau+1)}} \otimes \mathbf{1}_{2^{n}}^{\mathrm{T}}\right) F u(t) \Psi_{2^{n(\tau+1)}} z(t) \\
& =\left(I_{2^{n(\tau+1)}} \otimes \mathbf{1}_{2^{n}}^{\mathrm{T}}\right) F\left(I_{2^{r}} \otimes \Psi_{2^{n(\tau+1)}}\right) u(t) z(t) \\
& \triangleq L u(t) z(t),
\end{aligned}
$$

where $L=\left(I_{2^{n(\tau+1)}} \otimes \mathbf{1}_{2^{n}}^{\mathrm{T}}\right) F\left(I_{2^{r}} \otimes \Psi_{2^{n(\tau+1)}}\right) \in \mathcal{L}_{2^{n(\tau+1)} \times 2^{n(\tau+1)+r}}$.

In fact, the algebraic representation (7) on $x$ can also be obtained from the algebraic representation (20) on $z$. In the following, we will explain this fact.

Note that $x(t+1)=\left(I_{2^{n}} \otimes \mathbf{1}_{2^{n \tau}}^{\mathrm{T}}\right) z(t+1)$, then by the algebraic representation (20), one obtains the algebraic representation on $x$ as follows

$$
x(t+1)=\left(I_{2^{n}} \otimes \mathbf{1}_{2^{n \tau}}^{\mathrm{T}}\right) L u(t) x(t) \cdots x(t-\tau) .
$$

Our task now is to verify that

$$
\left(I_{2^{n}} \otimes \mathbf{1}_{2^{n \tau}}^{\mathrm{T}}\right) L=F .
$$

Applying the properties of the Kronecker product yields

$$
\begin{aligned}
\left(I_{2^{n}} \otimes \mathbf{1}_{2^{n \tau}}^{\mathrm{T}}\right) L & =\left(I_{2^{n}} \otimes \mathbf{1}_{2^{n \tau}}^{\mathrm{T}}\right)\left(I_{2^{n(\tau+1)}} \otimes \mathbf{1}_{2^{n}}^{\mathrm{T}}\right) F\left(I_{2^{r}} \otimes \Psi_{2^{n(\tau+1)}}\right) \\
& =\left\{I_{2^{n}} \otimes\left[\mathbf{1}_{2^{n \tau}}^{\mathrm{T}}\left(I_{2^{n \tau}} \otimes \mathbf{1}_{2^{n}}^{\mathrm{T}}\right)\right]\right\} F\left(I_{2^{r}} \otimes \Psi_{2^{n(\tau+1)}}\right) \\
& =\left(I_{2^{n}} \otimes \mathbf{1}_{2^{n(\tau+1)}}^{\mathrm{T}}\right)\left(F \otimes I_{2^{n(\tau+1)}}\right)\left(I_{2^{r}} \otimes \Psi_{2^{n(\tau+1)}}\right) \\
& =\left(F \otimes \mathbf{1}_{2^{n(\tau+1)}}^{\mathrm{T}}\right)\left(I_{2^{r}} \otimes \Psi_{2^{n(\tau+1)}}\right) .
\end{aligned}
$$

Since $F$ in (77) is a $2^{n} \times 2^{n(\tau+1)+r}$ logical matrix, we split it into $2^{r}$ square blocks as $F=\left[\begin{array}{llll}F_{1} & F_{2} & \cdots & F_{2^{r}}\end{array}\right]$, where $F_{k} \in \mathcal{L}_{2^{n} \times 2^{n(\tau+1)}}, k=1, \ldots, 2^{r}$. Then, we have

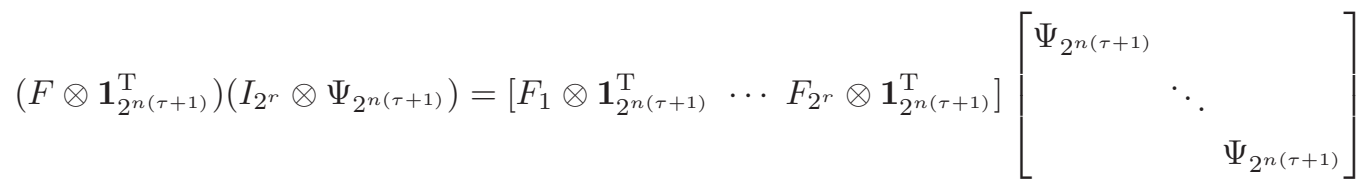

$$
\begin{aligned}
& =\left[\begin{array}{lll}
\left(F_{1} \otimes \mathbf{1}_{2^{n(\tau+1)}}^{\mathrm{T}}\right) \Psi_{2^{n(\tau+1)}} & \cdots\left(F_{2^{r}} \otimes \mathbf{1}_{2^{n(\tau+1)}}^{\mathrm{T}}\right) \Psi_{2^{n(\tau+1)}}
\end{array}\right] \\
& =\left[\begin{array}{lll}
F_{1} & \cdots & F_{2^{r}}
\end{array}\right] \text {. }
\end{aligned}
$$

This together with (23) implies that (22) holds.

According to the above analysis, one can conclude that there is a one-to-one and onto mapping between (17) and (20). Therefore, the reachability as well as controllability of ABCN (3) can be investigated directly from system (20) instead of system (7).

\subsection{Controllability of the ABCNs}

In what follows, the controllability of ABCNs (3) is addressed based on the algebraic form (20). We first present the definition of controllability for network (3).

Definition 3. Consider system (3). For any given initial time $t_{0}$, any given set of time delays $\left\{\tau_{i j} \in\right.$ $\mathcal{N}: i, j=1, \ldots, n\}$, any given initial state sequence $X_{0}=\left\{x\left(t_{0}-\tau\right), x\left(t_{0}-\tau+1\right), \ldots, x\left(t_{0}\right)\right\} \sim x_{0}=$ $\ltimes_{k=0}^{\tau} x\left(t_{0}-k\right) \in \Delta_{2^{n(\tau+1)}}$, any given destination state $x_{d} \in \Delta_{2^{n}}$, and any given $k \in \mathcal{N} \backslash\{0\}$, 
Chen H W, et al. Sci China Inf Sci January 2016 Vol. xx xxxxxx:9

1) $x_{d}$ is said to be reachable from $x_{0}$ at the $k$ th step if a control sequence $\left\{u\left(t_{0}\right), u\left(t_{0}+1\right), \ldots, u\left(t_{0}+\right.\right.$ $k-1)\}$ can be found such that the trajectory of (3) satisfies $x\left(t_{0}+k\right)=x_{d}$.

2) The set of all states that are reachable from $x_{0}$ at the $k$ th step is said to be the $k$-step reachable set of $x_{0}$, denoted by $R_{k}\left(x_{0}\right)$.

3) The set of all states that are reachable from $x_{0}$ is called to be the reachable set of $x_{0}$, denoted by $R\left(x_{0}\right)$. Clearly $R\left(x_{0}\right)=\cup_{k \in \mathcal{N} \backslash\{0\}} R_{k}\left(x_{0}\right)$.

4) System (3) is said to be controllable from $x_{0}$ if $R\left(x_{0}\right)=\Delta_{2^{n}}$.

5) System (3) is said to be (globally) controllable if it is controllable from any $x_{0} \in \Delta_{2^{n(\tau+1)}}$.

For $x_{0} \in \Delta_{2^{n(\tau+1)}}$ and $x_{d} \in \Delta_{2^{n}}$, let $l\left(k ; x_{0}, x_{d}\right)$ denote the number of different control sequences that steer ABCN (3) from $x_{0}$ to $x_{d}$ at the $k$ th time-step. The following result provides a simple algebraic expression for $l\left(k ; x_{0}, x_{d}\right)$.

Theorem 3. Consider system (3) with algebraic form (20). Let $l\left(k ; x_{0}, x_{d}\right)$ denote the number of different control sequences that steer ABCN (3) from $x_{0}=\delta_{2^{n(\tau+1)}}^{j}$ to $x_{d}=\delta_{2^{n}}^{i}$ at the $k$ th time-step. Then

$$
l\left(k ; x_{0}, x_{d}\right)=\left[\left(I_{2^{n}} \otimes \mathbf{1}_{2^{n \tau}}^{\mathrm{T}}\right) M^{k}\right]_{i j},
$$

where $M=L \mathbf{1}_{2^{r}}$.

Proof. We prove this result by induction. Consider firstly the case $k=1$. For a given initial state $x_{0}$, it follows from (21) that

$$
x_{d}=x\left(t_{0}+1\right)=\left(I_{2^{n}} \otimes \mathbf{1}_{2^{n \tau}}^{\mathrm{T}}\right) L \ltimes u\left(t_{0}\right) \ltimes x_{0} .
$$

Let $\mu^{1}\left(t_{0}\right), \ldots, \mu^{\alpha}\left(t_{0}\right)$ be the different control sequences steering (25) from $x_{0}$ to $x_{d}$, i.e.,

$$
x_{d}=\left(I_{2^{n}} \otimes \mathbf{1}_{2^{n \tau}}^{\mathrm{T}}\right) L \ltimes \mu^{i}\left(t_{0}\right) \ltimes x_{0}, \quad i \in\{1, \ldots, \alpha\} .
$$

Since each value of the controllers is a column of $I_{2^{r}}$, there exist $\beta=2^{r}-\alpha$ different control sequences $\left\{\nu^{j}\left(t_{0}\right)\right\}$ such that

$$
x_{d} \neq\left(I_{2^{n}} \otimes \mathbf{1}_{2^{n \tau}}^{\mathrm{T}}\right) L \ltimes \nu^{j}\left(t_{0}\right) \ltimes x_{0}, \quad j \in\{1, \ldots, \beta\} .
$$

Multiplying both sides of (26) and (27) with $x_{d}^{\mathrm{T}}$ yields

$$
\begin{aligned}
& 1=x_{d}^{\mathrm{T}}\left(I_{2^{n}} \otimes \mathbf{1}_{2^{n \tau}}^{\mathrm{T}}\right) L \ltimes \mu^{i}\left(t_{0}\right) \ltimes x_{0}, \quad i \in\{1, \ldots, \alpha\}, \\
& 0=x_{d}^{\mathrm{T}}\left(I_{2^{n}} \otimes \mathbf{1}_{2^{n \tau}}^{\mathrm{T}}\right) L \ltimes \nu^{j}\left(t_{0}\right) \ltimes x_{0}, \quad j \in\{1, \ldots, \beta\} .
\end{aligned}
$$

Summing up the above $2^{r}$ equations together, we have

$$
\begin{aligned}
\alpha & =x_{d}^{\mathrm{T}}\left(I_{2^{n}} \otimes \mathbf{1}_{2^{n \tau}}^{\mathrm{T}}\right) L \ltimes \mathbf{1}_{2^{r}} \ltimes x_{0} \\
& =x_{d}^{\mathrm{T}}\left(I_{2^{n}} \otimes \mathbf{1}_{2^{n \tau}}^{\mathrm{T}}\right) M x_{0} \\
& =\left[\left(I_{2^{n}} \otimes \mathbf{1}_{2^{n \tau}}^{\mathrm{T}}\right) M\right]_{i j},
\end{aligned}
$$

which means that (24) holds for $k=1$.

Secondly, assume that (24) holds for $k=n$. For the induction step, we consider finally the case of $k=n+1$. Note that the number of control sequences that steer system (3) from $x_{0}=z\left(t_{0}\right)$ to $x_{d}$ at $n+1$ time-step equals to the sum, over all possible states $z\left(t_{0}+1\right)=\delta_{2^{n(\tau+1)}}^{\lambda}$, of the product of (i) the number of control sequences that steer system (3) from $z\left(t_{0}\right)$ to $z\left(t_{0}+1\right)$ at one step; and (ii) the number of control sequences that steer system (3) from $z\left(t_{0}+1\right)$ to $x_{d}$ at $n$ steps. It thus follows that

$$
\begin{aligned}
& l\left(n+1 ; x_{0}, x_{d}\right)=\sum_{\lambda=1}^{2^{n(\tau+1)}} l\left(1 ; z\left(t_{0}\right), z\left(t_{0}+1\right)\right) l\left(n ; z\left(t_{0}+1\right), x_{d}\right) \\
& =\sum_{\lambda=1}^{2^{n(\tau+1)}} l\left(n ; z\left(t_{0}+1\right), x_{d}\right) l\left(1 ; z\left(t_{0}\right), z\left(t_{0}+1\right)\right) .
\end{aligned}
$$


Chen H W, et al. Sci China Inf Sci January 2016 Vol. xx xxxxxx:10

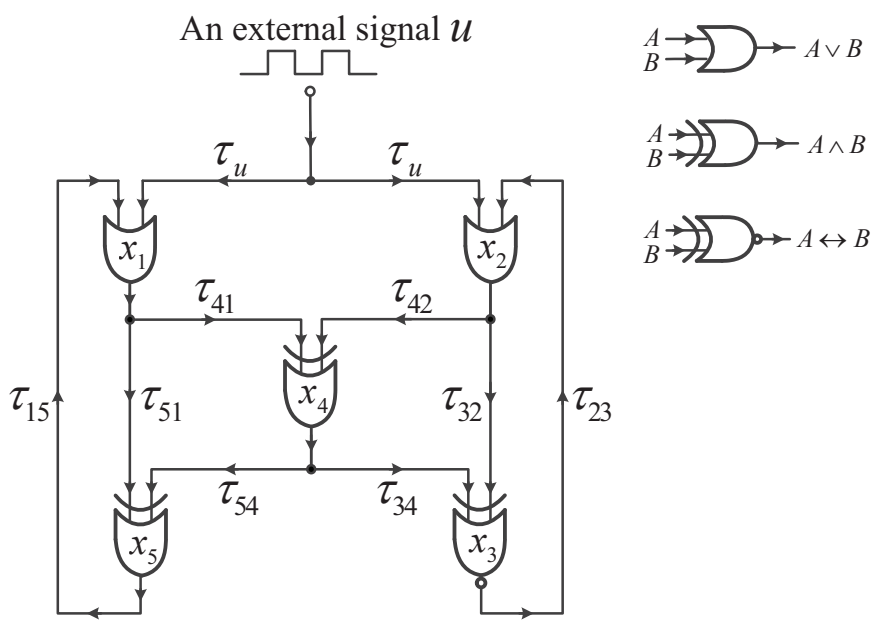

Figure 1 Schematic of the network topology for the five-node ABCN on the IEEE Std 91/91a-1991 representation [33].

Applying the induction hypothesis yields

$$
\begin{aligned}
l\left(n+1 ; x_{0}, x_{d}\right) & =\sum_{\lambda=1}^{2^{n(\tau+1)}} x_{d}^{\mathrm{T}}\left[\left(I_{2^{n}} \otimes \mathbf{1}_{2^{n \tau}}^{\mathrm{T}}\right) M^{n}\right] z\left(t_{0}+1\right) z\left(t_{0}+1\right)^{\mathrm{T}} M z\left(t_{0}\right) \\
& =\sum_{\lambda=1}^{2^{n(\tau+1)}}\left[\left(I_{2^{n}} \otimes \mathbf{1}_{2^{n \tau}}^{\mathrm{T}}\right) M^{n}\right]_{i \lambda} M_{\lambda j} \\
& =\left[\left(I_{2^{n}} \otimes \mathbf{1}_{2^{n \tau}}^{\mathrm{T}}\right) M^{n+1}\right]_{i j},
\end{aligned}
$$

which infers that (24) holds for $k=n+1$. By induction, one can conclude that (24) holds for any positive integer $k$. The proof is then complete.

In fact, the $k$ in Definition 3 about reachability and controllability depends on both $x_{0}$ and $x_{d}$. Note that the cardinal number of the state space $\Delta_{2^{n(\tau+1)}}$ is $2^{n(\tau+1)}$, one can choose $k\left(x_{0}, x_{d}\right) \leqslant 2^{n(\tau+1)}-1:=N$. Based on Theorem 3, the following result on testing the controllability of (3) is obtained.

Theorem 4. Consider system (3) with algebraic form (20). Let $N=2^{n(\tau+1)}-1$, then

1) $x_{d}=\delta_{2^{n}}^{i}$ is reachable from $x_{0}=\delta_{2^{n(\tau+1)}}^{j}$ at the $k$ th step if and only if

$$
\left[\left(I_{2^{n}} \otimes \mathbf{1}_{2^{n \tau}}^{\mathrm{T}}\right) M^{k}\right]_{i j}>0 .
$$

2) System (3) is controllable from $x_{0}=\delta_{2^{n(\tau+1)}}^{j}$ if and only if

$$
\operatorname{Col}_{j}\left(\left(I_{2^{n}} \otimes \mathbf{1}_{2^{n \tau}}^{\mathrm{T}}\right) \sum_{\lambda=1}^{N} M^{\lambda}\right)>0 .
$$

3) System (3) is controllable if and only if

$$
\left(I_{2^{n}} \otimes \mathbf{1}_{2^{n \tau}}^{\mathrm{T}}\right) \sum_{\lambda=1}^{N} M^{\lambda}>0 .
$$

Remark 3. Theorem 4 provides some necessary and sufficiency conditions for the reachability and controllability of $\mathrm{ABCN}$ (3) with pinning controllers, and the obtained matrix testing criteria imply that one only need to check for a finite number of $N$. One can observe that the matrix $M$ defined in Theorem 3 is a $2^{n(\tau+1)} \times 2^{n(\tau+1)}$ matrix, which means that the dimensions of $M$ grows exponentially as the size of the network (3) increases. Thus, the proposed criteria are applicable only to the small scale of ABCNs. 
Example 3. In 33, the authors have proposed the topology of an ABN that is driven by an external signal $u$ as shown in Figure 1. The system is an electronic circuit that realizes the Boolean nodes with logic gates, specifically, nodes 1 and 2 execute the OR logic operation, node 3 executes the XNOR logic operation, while nodes 4 and 5 execute the XOR logic operation. The time that it takes a signal to propagate to node $i$ from node $j$ is denoted by $\tau_{i j}(1 \leqslant i, j \leqslant 5)$. Each time delay comes about from a combination of an intrinsic delay associated with each gate and the signal propagation time along the connecting links. The Boolean delay equations describing this ABCN are as follows:

$$
\left\{\begin{array}{l}
x_{1}(t)=u\left(t-\tau_{u}\right) \vee x_{5}\left(t-\tau_{15}\right), \\
x_{2}(t)=u\left(t-\tau_{u}\right) \vee x_{3}\left(t-\tau_{23}\right), \\
x_{3}(t)=x_{2}\left(t-\tau_{32}\right) \leftrightarrow x_{4}\left(t-\tau_{34}\right), \\
x_{4}(t)=x_{1}\left(t-\tau_{41}\right) \wedge x_{2}\left(t-\tau_{42}\right), \\
x_{5}(t)=x_{1}\left(t-\tau_{51}\right) \wedge x_{4}\left(t-\tau_{54}\right),
\end{array}\right.
$$

where the values of $\tau_{i j}$ are given in Table 1.

Table 1 Time delays $\tau_{i j}$ in system 31.

\begin{tabular}{ccccccccc}
\hline$\tau_{u}$ & $\tau_{15}$ & $\tau_{23}$ & $\tau_{32}$ & $\tau_{34}$ & $\tau_{41}$ & $\tau_{42}$ & $\tau_{51}$ & $\tau_{54}$ \\
\hline 1 & 2 & 1 & 1 & 1 & 2 & 2 & 1 & 2 \\
\hline
\end{tabular}

Let $x=\ltimes_{k=1}^{5} x_{k}$, then we can express the ABCN (31) in its algebraic form as

$$
x(t+1)=F u(t) x(t) x(t-1),
$$

where $F$ is a $2^{5} \times 2^{11}$ logical matrix which is omitted here for space consideration, and $t=0,1,2, \ldots$. Set $z_{0}(t)=x(t)$ and $z_{1}(t)=x(t-1)$, the equivalent algebraic form of ABCN (32) can be obtained as $z(t+1)=L u(t) z(t)$, where $L \in \mathcal{L}_{2^{10} \times 2^{11}}$. In order to check whether or not the ABCN (31) is controllable, we should calculate

$$
\left(I_{2^{n}} \otimes \mathbf{1}_{2^{n \tau}}^{\mathrm{T}}\right) \sum_{\lambda=1}^{2^{10}-1} M^{\lambda}:=\left[\Gamma_{1} \Gamma_{2} \Gamma_{3} \Gamma_{4}\right] \in \mathbb{R}^{32 \times 1024}
$$

according to Theorem 4. By choosing $\bar{N}=20$, and replacing the nonzero entries of $\Gamma_{k} \in \mathbb{R}^{32 \times 256}$ $(k=1, \ldots, 4)$ by 1 , we obtain

$$
\Gamma_{1}=\Gamma_{2}=\Gamma_{3}=\Gamma_{4}>0,
$$

and Figure 2 plot the whole row indexes of each column of matrix $\Gamma_{k}$. Thus, one can conclude that the $\mathrm{ABCN}$ (31) is globally controllable.

\subsection{Pinning control design algorithm}

Consider the problem of designing a pinning control sequence that steers the ABCN (3) between any two given states $x_{0}$ and $x_{d}$, which seems relevant to the therapeutic intervention, since some states may correspond to the diseased states.

First, for system (20), if the two states $z_{d}=\delta_{2^{n(\tau+1)}}^{i^{\prime}}$ and $z\left(t_{0}\right)=\delta_{2^{n(\tau+1)}}^{j}$ satisfy $\delta_{2^{n(\tau+1)}}^{i^{\prime}} \in R\left(\delta_{2^{n(\tau+1)}}^{j}\right)$, Algorithm 1 can generate any control sequence steering $z\left(t_{0}\right)$ to $z_{d}$.

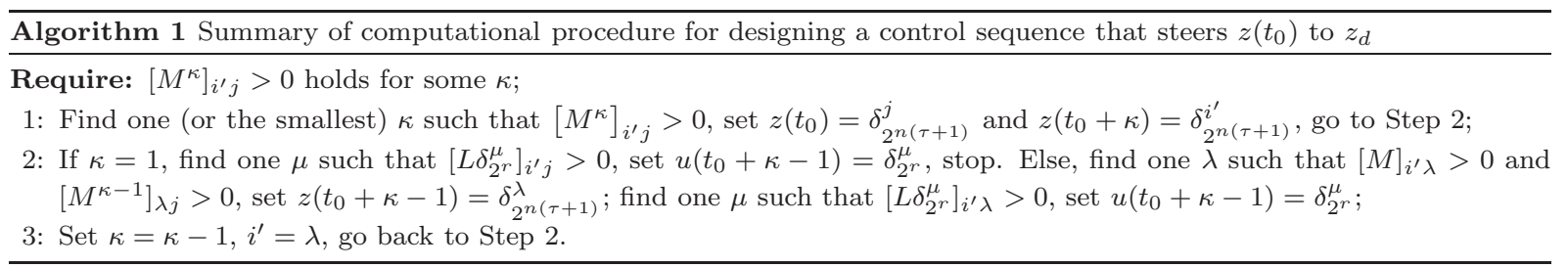

Next, consider ABCN (3) with its algebraic form (20). Suppose that the two given states $x_{0}=\delta_{2^{n(\tau+1)}}^{j}$ and $x_{d}=\delta_{2^{n}}^{i}$ satisfying $x_{d} \in R\left(x_{0}\right)$, then by Theorem 4 , one has

$$
\left[\left(I_{2^{n}} \otimes \mathbf{1}_{2^{n \tau}}^{\mathrm{T}}\right) M^{\kappa}\right]_{i j}>0
$$


Chen H W, et al. Sci China Inf Sci January 2016 Vol. xx xxxxxx:12
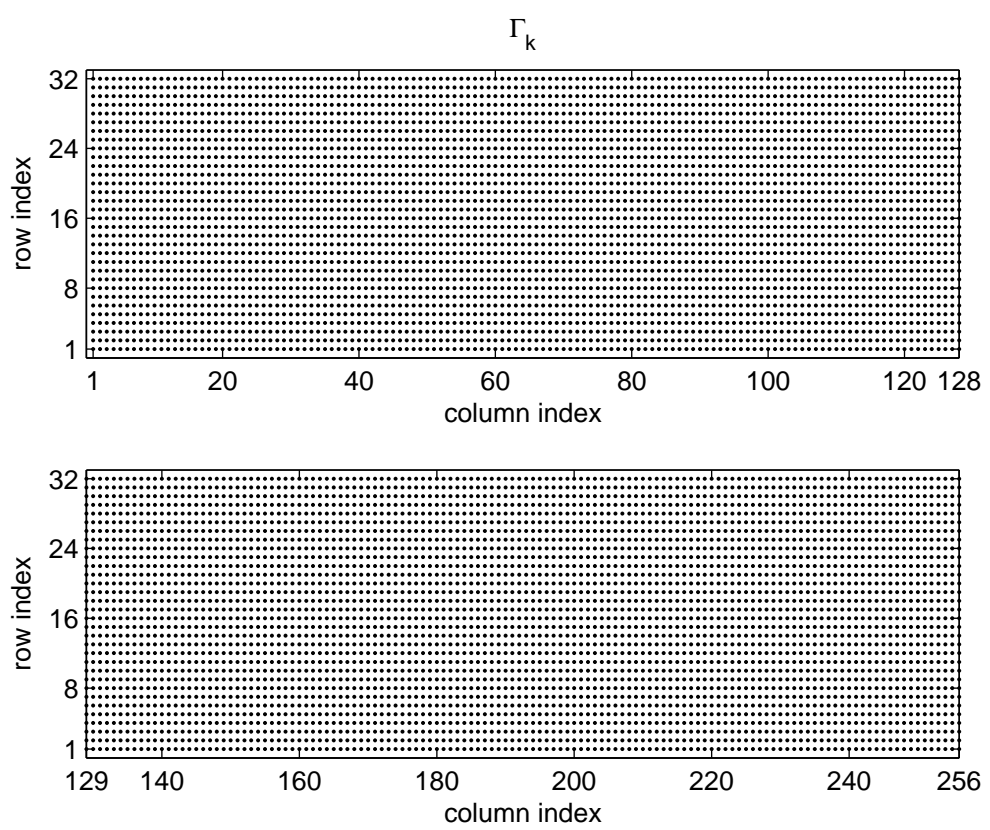

Figure 2 The whole row indexes of each column of matrix $\Gamma_{k}$. Each point corresponds to the row index of each column, which implies the position of 1 .

holds for some $\kappa$, which implies that

$$
\operatorname{Row}_{i}\left(I_{2^{n}} \otimes \mathbf{1}_{2^{n \tau}}^{\mathrm{T}}\right) \operatorname{Col}_{j}\left(M^{\kappa}\right)>0 .
$$

Thus, there exists at least one $i^{\prime} \in\left\{(i-1) 2^{n \tau}+l: l=1,2, \ldots, 2^{n \tau}\right\}$ such that $\left[M^{\kappa}\right]_{i^{\prime} j}>0$, and we have

$$
\delta_{2^{n}}^{i}=\left(I_{2^{n}} \otimes \mathbf{1}_{2^{n \tau}}^{\mathrm{T}}\right) \delta_{2^{n(\tau+1)}}^{i^{\prime}} .
$$

Then, by Algorithm 1, one can find a control sequence $\left\{u\left(t_{0}\right), u\left(t_{0}+1\right), \ldots, u\left(t_{0}+k-1\right)\right\}$ steering $\delta_{2^{n(\tau+1)}}^{j}$ to $\delta_{2^{n(\tau+1)}}^{i^{\prime}}$, thereby providing the pinning control sequence steering the ABCN (3) from $x_{0}=\delta_{2^{n(\tau+1)}}^{j}$ to $x_{d}=\delta_{2^{n}}^{i}$.

Finally, based on the above analysis, we give the following pinning control design algorithm for system (3). It is worth pointing out that the pinning controller form $x_{0}$ to $x_{d}$ is generally not unique, Algorithm2 gives a unified method to find all pinning controllers, including the shortest ones.

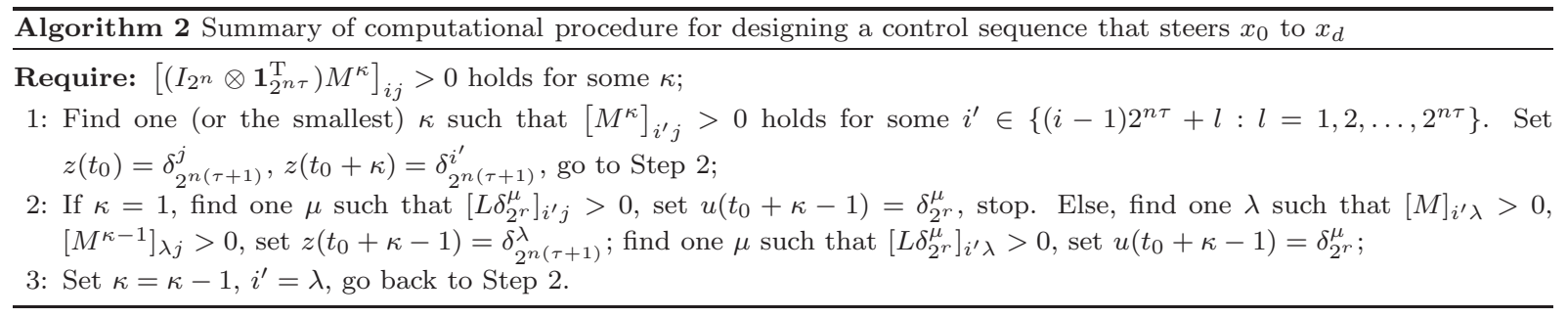

Remark 4. The time delays in ABCN (3) are all assumed to be constant, and it is possible to extend the present study to the case of time-varying delays by splitting the system into a finite number of subsystems with no time delays, called the constructed forest. The readers are referred to [26] for more details.

Example 4. Consider the ABCN in Example 3, and design a control sequence to steer the initial state $x_{0}=\delta_{1024}^{242}$ to the destination state $x_{d}=\delta_{32}^{19}$ (if possible). We follow Algorithm 2 step by step as follows:

- Step 1. A calculation yields $\left[M^{3}\right]_{603,242}>0$ with $l=27$. Set $z(0)=\delta_{1024}^{242}, z(3)=\delta_{1024}^{603}$.

- Step 2. From a straightforward computation, one gets $[M]_{603,838}>0,\left[M^{2}\right]_{838,242}>0$, set $z(2)=$ $\delta_{1024}^{838}$. One also gets $\left[L \delta_{2}^{2}\right]_{603,838}>0$, set $u(2)=\delta_{2}^{2}$. 
- Step 3. From a straightforward computation, one gets $[M]_{838,168}>0,\left[M^{1}\right]_{168,242}>0$, set $z(1)=$ $\delta_{1024}^{168}$. One also gets $\left[L \delta_{2}^{2}\right]_{838,168}>0$, set $u(1)=\delta_{2}^{2}$.

- Step 4. From a straightforward computation, one gets $\left[L \delta_{2}^{1}\right]_{168,242}>0$, set $u(0)=\delta_{2}^{1}$.

Consequently, one obtains the control sequence $\left\{u(0)=\delta_{2}^{1}, u(1)=\delta_{2}^{2}, u(2)=\delta_{2}^{2}\right\}$ steering ABCN (31) from $x_{0}=\delta_{1024}^{242}$ to $x_{d}=\delta_{32}^{19}$ at the third step. Since $z(1)=\delta_{1024}^{168}, z(2)=\delta_{1024}^{838}$, the corresponding state trajectory is

$$
\left\{\begin{array}{r}
x(0)=\delta_{32}^{8} \\
x(-1)=\delta_{32}^{18}
\end{array}\right\} \stackrel{u(0)=\delta_{2}^{1}}{\longrightarrow} x(1)=\delta_{32}^{6} \stackrel{u(1)=\delta_{2}^{2}}{\longrightarrow} x(2)=\delta_{32}^{27} \stackrel{u(2)=\delta_{2}^{2}}{\longrightarrow} x(3)=\delta_{32}^{19} .
$$

\section{Conclusion}

In this paper, the pinning controllability of the $\mathrm{ABCNs}$ has been studied, and several new results have been presented. By resorting to the STP technique, ABCNs with pinning controllers have been converted into the algebraic form, and some inherent special structures of the network transition matrix have been characterized. Then, a simple algebraic formula has been obtained for the number of different control sequences that steer an $\mathrm{ABCN}$ from the given initial state to the desired terminal state at a given number of time step. With this expression, some computable algebraic criteria have been derived for the pinning controllability of the ABCNs. Moreover, a practical control method has been devised that can be implemented to only a fraction of nodes to force the whole system to the desired state. A practical ABCN realized by logic circuits has been given to highlight the utility of the obtained results.

On the other hand, the limitation of our method lies in the fact that the state space grows exponentially as the size of the network increases. Further research is needed to apply the obtained results to the largescale ABCNs. Another interesting yet difficult task in future work is to determine the potential nodes selected to be controlled. In summary, the proposed approach provides new insights on understanding and controlling the dynamics of GRNs by means of ABCNs, and it also has implications for the therapeutic intervention and in the genetic engineering.

Acknowledgements This work was supported in part by the National Natural Science Foundation of China under Grant 61329301 and 61273156, the Natural Science Foundation of Jiangsu Province of China under Grant BK20130017, the Six Talent Peaks Project for the High Level Personnel from the Jiangsu Province of China under Grant 2015-DZXX-003, and the Scientific Research Foundations of Graduate School of Southeast University and Innovation Program of Jiangsu Province (No. KYZZ15_0050).

Conflict of interest The authors declare that they have no conflict of interest.

\section{References}

1 Wang $\mathrm{Z}, \mathrm{Wu} \mathrm{H}$, Liang $\mathrm{J}$, et al. On modeling and state estimation for genetic regulatory networks with polytopic uncertainties. IEEE Trans Nanobiosci, 2013, 12: 13-20

2 Shen B, Wang Z, Liang J, et al. Sampled-data $H_{\infty}$ filtering for stochastic genetic regulatory networks. Int J Robust Nonlinear Control, 2011, 21: 1759-1777

3 Kauffman S A. Metabolic stability and epigenesis in randomly constructed genetic nets. J Theor Biol, 1969, 22: 437-467

4 Aldana M. Boolean dynamics of networks with scale-free topology. Physica D, 2003, 185: 45-66

5 Akutsu T, Hayashida M, Ching W-K, et al. Control of Boolean networks: hardness results and algorithms for tree structured networks. J Theor Biol, 2007, 244: 670-679

6 Ideker T, Galitski T, Hood L. A new approach to decoding life: systems biology. Annu Rev Genomics Hum Genet, 2001, 2: 343-372

7 Cheng D, Qi H. A linear representation of dynamics of Boolean networks. IEEE Trans Autom Control, 2010, 55: $2251-2258$

8 Cheng D, Qi H. Controllability and observability of Boolean control networks. Automatica, 2009, 45: 1659-1667

9 Laschov D, Margaliot M. Controllability of Boolean control networks via the Perron-Frobenius theory. Automatica, 2012, 48: 1218-1223

10 Fornasini E, Valcher M E. Observability, reconstructibility and state observers of Boolean control networks. IEEE Trans Autom Control, 2013, 58: 1390-1401

11 Cheng D, Qi H, Li Z, et al. Stability and stabilization of Boolean networks. Int J Robust Nonlinear Control, 2011, 21: 134-156

12 Li H, Wang Y. Consistent stabilizability of switched Boolean networks. Neural Netw, 2013, 46: 183-189 
Chen H W, et al. Sci China Inf Sci January 2016 Vol. xx xxxxxx:14

13 Li H, Wang Y, Liu Z. Stability analysis for switched Boolean networks under arbitrary switching signals. IEEE Trans Autom Control, 2014, 59: 1978-1982

14 Zhao Y, Cheng D. On controllability and stabilizability of probabilistic Boolean control networks. Sci China-Inf Sci, 2014, 57: 012202

15 Fornasini E, Valcher M E. Optimal control of Boolean control networks. IEEE Trans Autom Control, 2014, 59: $1258-1270$

16 Laschov D, Margaliot M. A maximum principle for single-input Boolean control networks. IEEE Trans Autom Control, 2011, 56: 913-917

17 Zou Y, Zhu J. System decomposition with respect to inputs for Boolean control networks. Automatica, 2014, 50: 1304-1309

18 Zou Y, Zhu J. Kalman decomposition for Boolean control networks. Automatica, 2015, 54: 65-71

19 Cheng D. On finite potential games. Automatica, 2014, 50: 1793-1801

$20 \mathrm{Xu} \mathrm{X}$, Hong Y. Matrix approach to model matching of asynchronous sequential machines. IEEE Trans Autom Control, 2013, 58: 2974-2979

21 Smolen P, Baxter D A, Byrne J H. Mathematical modeling of gene networks. Neuron, 2000, 26: 567-580

22 Li F, Sun J. Controllability of Boolean control networks with time delays in states. Automatica, 2011, 47: 603-607

23 Li F, Sun J. Controllability of higher order Boolean control networks. Appl Math Comput, 2012, 219: 158-169

24 Chen H, Sun J. A new approach for global controllability of higher order Boolean control network. Neural Netw, 2013, 39: $12-17$

25 Zhang L, Zhang K. Controllability of time-variant Boolean control networks and its application to Boolean control networks with finite memories. Sci China-Inf Sci, 2013, 56: 108201

26 Zhang L, Zhang K. Controllability and observability of Boolean control networks with time-variant delays in states. IEEE Trans Neural Netw Learn Syst, 2013, 24: 1478-1484

27 Müller F-J, Schuppert A. Few inputs can reprogram biological networks. Nature, 2011, 478: E4-E4

28 Lin G-Q, Ao B, Chen J-W, et al. Modeling and controlling the two-phase dynamics of the p53 network: a Boolean network approach. New J Phys, 2014, 16: 125010

29 Lu J, Zhong J, Huang C, et al. On pinning controllability of Boolean control networks. IEEE Trans Autom Control, DOI:10.1109/TAC.2015.2478123, in press

30 Li F. Pinning control design for the stabilization of Boolean networks. IEEE Trans Neural Netw Learn Syst, DOI: 10.1109/TNNLS.2015.2449274, in press

31 Ghil M, Zaliapin I, Coluzzi B. Boolean delay equations: a simple way of looking at complex systems. Physica D, 2008, 237: $2967-2986$

32 Zhang R, Cavalcante H L, Gao Z, et al. Boolean chaos. Phys Rev E, 2009, 80: 045202

33 Rivera-Durón R R, Campos-Cantón E, Campos-Cantón I, et al. Forced synchronization of autonomous dynamical Boolean networks. Chaos, 2015, 25: 083113

34 Glass L, Perkins T J, Mason J, et al. Chaotic dynamics in an electronic model of a genetic network. J Stat Phys, 2005, 121: 969-994

35 Cheng X, Sun M, Socolar J E S. Autonomous Boolean modelling of developmental gene regulatory networks. J R Soc Interface, 2013, 10: 20120574 